\title{
Pegada hídrica de plantas hortícolas cultivadas no semiárido brasileiro
}

A atual crise hídrica está relacionada a diversas ações antropogênicas no planeta, entre elas, a utilização demasiada da água no setor agrícola. A Pegada Hídrica (PH) é um indicador da apropriação de recursos hídricos que quantifica o uso direto e indireto deste recurso, possuindo o intuito de subsidiar o estabelecimento de políticas e ações concretas para economia de água. O objetivo desta pesquisa é determinar as pegadas hídricas de culturas do tomate (Solanum lycopersicum), cebola (Allium cepa), feijão (Phaseolus vulgaris), alface (Lactuca sativa L.) e o coentro (Coriandrum sativum). A parte experimental do estudo foi realizado na Estação Agrometeorológica Experimental (EstAgro - DCA da Universidade Federal de Campina Grande) e foram determinadas a PH de cada cultura com base na evapotranspiração das culturas determinadas pelo método do balanço hídrico do solo e pelo software Cropwat. A evapotranspiração de referência (ETo) fo determinada com base no método de Penman-Monteith. Os resultados deste trabalho permitem concluir que as hortaliças folhosas têm alto componente da PH cinza comparada com os componentes verde e azul da cultura. As pegadas hídricas azul e verde aumentam de acordo com o aumento da irrigação e precipitação pluvial, porém a pegada hídrica cinza varia apenas em função da produtividade.

Palavras-chave: Evapotranspiração; Produtividade; Modelo Cropwat.

\section{Water footprint of vegetables grown in the Brazilian semiarid}

\begin{abstract}
The current water crisis is related to several anthropogenic actions on the planet, including the overuse of water in the agricultural sector. The Water Footprint $(\mathrm{PH})$ is an indicator of the appropriation of water resources that quantifies the direct and indirect use of this resource, aiming to subsidize the establishment of policies and concrete actions for water saving. The objective of this research is to determine the water footprints of tomato (Solanum lycopersicum), onion (Allium cepa), beans (Phaseolus vulgaris), lettuce (Lactuca sativa L.) and coriander (Coriandrum sativum) crops. The experimental part of the study was carried out at the Experimental Agrometeorological Station (EstAgro - DCA of the Federal University of Campina Grande) and the pH of each crop was determined based on the crop evapotranspiration determined by the soil water balance method and Cropwat software. Reference evapotranspiration (ETo) was determined based on the Penman-Monteith method. The results of this work allow us to conclude that leafy vegetables have a high component of gray PH compared to green and blue components of the crop. Blue and green water footprints increase with increasing irrigation and rainfall, but the gray water footprint varies only as a function of productivity.
\end{abstract}

Keywords: Evapotranspiration; Productivity; Cropwat model.

Topic: Experimentação Agrícola

Reviewed anonymously in the process of blind peer.
Received: 05/06/2019

Approved: 06/07/2019
Rafaela Silveira Rodrigues Almeida (iD Universidade Federal de Campina Grande, Brasil http://lattes.cnpq.br/9716505205420143 http://orcid.org/0000-0002-1950-8029 rafasilver@ymail.com

\section{Madson Tavares Silva}

Universidade Federal de Campina Grande, Brasil http://lattes.cnpq.br/8829792848536805

http://orcid.org/0000-0003-1823-2742 madson.geotech@gmail.com

João Hugo Baracuy da Cunha Campos (iD Universidade Estadual da Paraíba, Brasil http://lattes.cnpq.br/2677113447182816 http://orcid.org/0000-0002-4796-0696 ihugocampos@yahoo.com.br
Romildo Morant de Holanda (iD)

Universidade Federal Rural de Pernambuco, Brasil http://lattes.cnpq.br/4283130448063981 http://orcid.org/0000-0001-7945-3616 incolneloi@yahoo.com.br

José Dantas Neto (iD

Universidade Federal de Campina Grande, Brasil http://lattes.cnpq.br/9137226205129315

http://orcid.org/0000-0003-0798-6717 zedantas1955@gmail.com

\section{Enio Pereira de Souza (iD)}

Universidade Federal de Campina Grande, Brasil http://lattes.cnpq.br/7752212981363912

http://orcid.org/0000-0002-5095-0085 enio.souza@ufcg.edu.br
Wendy Kaori Matsunaga (iD

Universidade Federal de Campina Grande, Brasil http://lattes.cnpq.br/0256880651038969 http://orcid.org/0000-0002-6778-5450 wendymatsunaga1@gmail.com

Kamila Souza Santos

Universidade Federal de Campina Grande, Brasil http://lattes.cnpq.br/4169930363028154 http://orcid.org/0000-0002-0580-277X kamilasouza182@gmail.com

Vicente de Paulo Rodrigues da Silva (iD Universidade Federal de Campina Grande, Brasil http://lattes.cnpq.br/3750344965379269 http://orcid.org/0000-0003-4914-4833 vicente.paulo@ufcg.edu.br d

DOI: 10.6008/CBPC2179-6858.2019.004.0005
Referencing this:

ALMEIDA, R. S. R.; SILVA, M. T.; CAMPOS J. H. B. C.; HOLANDA, R. M.; DANTAS NETO, J.; MATSUNAGA, W. K.; SILVA, V. P. R.. Pegada hídrica de plantas hortícolas cultivadas no semiárido brasileiro. Revista lberoAmericana de Ciências Ambientais, v.10, n.4, p.45-56, 2019. DOI: http://doi.org/10.6008/CBPC2179-6858.2019.004.0005 


\section{INTRODUÇÃO}

O conceito de Pegada Hídrica (PH) proposto por Hoekstra et al. (2011) está sendo utilizado como um indicador da apropriação de recursos hídricos. A PH possui caráter multidimensional em termos de quantificar o volume de consumo direto ou indireto da água e contabilizar os volumes de poluição pelo tipo de atividade (SILVA et al., 2013). A PH é subdividida em Pegada Hídrica Verde (PH verde), Pegada Hídrica Azul (PH azul) e Pegada Hídrica Cinza (PH cinza). A PH verde considera o total de água precipitada que não escoa ou não repõe a água subterrânea, mas é armazenada no solo e utilizada pelas plantas nos processos evapotranspiratórios.

A PH azul é a água doce superficial ou subterrânea que é utilizada em cultivos irrigados. Já a PH cinza é a contabilização do volume de água necessário para depurar efluentes (MEKONNEN et al., 2011; TRAURING et al., 2018). A PH já é uma ferramenta consolidada em áreas de cultivos no mundo todo, como por exemplo, estimativas de PH foram desenvolvidas para café e chá holandês (CHAPAGAIN et al., 2008), para as culturas de grãos da Coreia (YOO et al., 2012), trigo (MEKONNEN et al., 2010; ABABAEl et al., 2014); milho, soja e trigo (ALDAYA et al., 2010), diversas culturas irrigadas no Sudão (AHMED et al., 2011), Espanha (ALDAYA et al., 2010); Itália (NANA et al., 2014; LAMASTRA et al., 2014), África do Sul (DABROWSKI et al., 2009) e Tailândia (GHEEWALA et al., 2014).

Em relação ao fracionamento dos tipos de consumo, as consideradas águas verde e azul no globo variam muito no tempo e no espaço. Em países asiáticos, como Índia, Indonésia, Vietnã, Tailândia, Mianmar e Filipinas, a fração da pegada hídrica verde é substancialmente maior que a pegada hídrica azul, enquanto nos EUA e Paquistão, a pegada hídrica azul é quatro vezes maior que a componente verde (MEKONNEN et al., 2012).

No entanto, numa perspectiva global, a pegada hídrica da produção agrícola no período de 19962005 foi de $7404 \mathrm{Gm}^{3} /$ ano- $^{1}$, sendo $78 \%$ verde, azul $12 \%$, e $10 \%$ de cinza. O trigo apresentou a maior participação no consumo do volume total, com 1.087G $\mathrm{G}^{3} \mathrm{ano}^{-1}$ (70\% verde, 19\% azul e cinza $11 \%$ ), seguido do arroz $\left(992 \mathrm{Gm}^{3} \mathrm{ano}^{-1}\right)$ e do milho $\left(770 \mathrm{Gm}^{3} \mathrm{ano}^{-1}\right)$. De outra forma, a palma e o algodão foram principais culturas com o menor consumo total de água (CHAPAGAIN et al., 2008; MEKONNEN et al., 2011). Embora os estudos quantifiquem várias PHs de países europeus, asiáticos e africanos, há escassez na literatura de estimar PH de culturas comerciais nos países da América Latina (ALDAYA et al., 2010).

O semiárido brasileiro apresenta escassez física e econômica da água. Nessas regiões, a exiguidade hídrica está associada a uma complexa sinergia de fatores, onde a agricultura deve ter por objeto a obtenção de rendimentos máximos por unidade de água aplicada, rendimento máximo por unidade de área cultivada, ou ainda, por unidade de custo (máxima produtividade econômica da cultura).

Entretanto, alguns poucos estudos abordando a melhoria e a compreensão da eficiência do uso da água com a utilização da PH são encontrados na literatura, tais como os de Rocha et al. (2014) que avaliou a pegada hídrica de diversas culturas no estado de Pernambuco; Ribeiro (2014) contabilizou a PH de Mangifera indica (manga) no submédido do vale do São Francisco; Santiago et al. (2017) observou a PH de Saccharum 
officinarum (cana de açúcar) em Corouripe (AL); Bomfim (2017) quantificou a PH do Coriandrum sativum (coentro) Itabaiana (SE) e Silva et al. (2015) que mediu e modelou a PH também de Saccharum officinarum (cana de açúcar), sendo ainda incipientes estes estudos com muitas outras culturas.

O conceito econômico da água segue o princípio da teoria da produção, em que cada nível de água aplicado às culturas deve corresponder a um ponto de produtividade, no qual o produtor fixa a quantidade de água a aplicar segundo um critério de racionalidade econômica (GOMES et al., 2004; HAROU et al., 2009; LEVIDOW et al., 2014). Neste sentido, uma pesquisa mais aprofundada sobre os diversos aspectos das necessidades hídricas de culturas comumente cultivadas na área semiárida brasileira é de grande importância para permitir o estabelecimento de políticas e ações concretas para a sustentabilidade da água na agricultura destas zonas. Assim, o objetivo principal deste estudo é determinar os componentes da pegada hídrica de cinco culturas cultivadas irrigadas nas condições edafoclimáticas do semiárido brasileiro.

\section{MATERIAL E MÉTODOS}

\section{Área de estudo e cálculo da evapotranspiração de referência}

Os dados deste estudo foram obtidos na Estação Agrometeorológica Experimental (EstAgro - DCA), com latitude sul de $7^{\circ} 12^{\prime} 52,85^{\prime \prime}$ e longitude oeste $35^{\circ} 54^{\prime} 26,78^{\prime \prime}$, da Universidade Federal de Campina Grande (UFCG), localizada na Microrregião Campina Grande e no Estado da Paraíba. A evapotranspiração de referência (ETo) foi obtida pelo método Penman-Monteith, e é definida pela seguinte função: $\mathrm{ETo}=\frac{0,408 \Delta(\mathrm{Rn}-\mathrm{G})+\gamma_{T+2} \frac{900}{3} \mathrm{u} 2\left(\mathrm{e}_{s}-e_{a}\right)}{\Delta+\gamma\left(1+0,34 u_{2}\right)}$, em que: ETo - evapotranspiração de referência $\left(\mathrm{mm} \mathrm{d}^{-1}\right) ; \mathrm{R}_{\mathrm{n}}$ - saldo de radiação à superfície da cultura $\left(\mathrm{MJ} \mathrm{m} \mathrm{m}^{-2} \mathrm{~d}^{-1}\right) ; \mathrm{G}$ - densidade do fluxo de calor do solo $\left(\mathrm{MJ} \mathrm{m}^{-2} \mathrm{~d}^{-1}\right)$; Ttemperatura do ar a $2 \mathrm{~m}$ de altura $\left({ }^{\circ} \mathrm{C}\right) ; \mathrm{u}_{2}$ - velocidade do vento a $2 \mathrm{~m}$ de altura $\left(\mathrm{m} \mathrm{s}^{-1}\right) ; \mathrm{e}_{\mathrm{s}^{-}}$pressão de vapor de saturação $(\mathrm{kPa})$; e e: pressão parcial de vapor $(\mathrm{kPa})$; $\Delta$ : declividade da curva de pressão de vapor de saturação $\left(\mathrm{KPa}{ }^{\circ} \mathrm{C}^{-1}\right)$; e $\mathrm{Y}$ - coeficiente psicométrico $\left(\mathrm{kPa}{ }^{\circ} \mathrm{C}^{-1}\right)$.

\section{Delineamento experimental e Cálculo da Evapotranspiração da cultura}

O experimento foi conduzido em blocos casualizados, com cinco culturas: o tomate (Solanum lycopersicum), a cebola (Allium cepa), o feijão (Phaseolus vulgaris) e o coentro (Coriandrum sativum) e o alface (Lactuca sativa L.), quatro lâminas de irrigação $(25,50$, 75 e $100 \%$ da ETo e com duas repetições, em um esquema fatorial $4 \times 4 \times 2$ e 3-4, com aplicações semanais de água até as senescências das culturas. Os componentes da equação do balanço hídrico para um volume de solo e controle do perfil até a profundidade da zona de raiz foram estimados a partir da seguinte equação: $E T_{c}=P+I+C_{p}+D_{p}-R_{o} \pm \Delta W$, em que $P$ é a precipitação $(\mathrm{mm})$; l é a irrigação $(\mathrm{mm})$; Cp é a ascensão capilar $(\mathrm{mm})$; Dp é a percolação de água na zona de raiz, também conhecida como drenagem profunda $(\mathrm{mm})$; Ro é o escoamento superficial $(\mathrm{mm})$; e W é a variação no conteúdo de água do solo $(\mathrm{mm})$.

O escoamento superficial $(R)$ e o fluxo a ascensão capilar para a zona de raiz $(\mathrm{C} p)$ serão considerados nulos, haja vista que eles são muito pequenos e assim desprezíveis, quando comparados com a $\mathrm{ET}_{c}$. Além 
disso, a drenagem profunda (Dp), pela mesma razão, será também considerada nula. Assim, a Eq. (3) é reduzida para: $\mathrm{ET}_{\mathrm{c}}=\mathrm{P}+\mathrm{I} \pm \Delta \mathrm{W}$. Quando os lisímetros não são instalados para o monitoramento da $\mathrm{ET}_{\mathrm{c}}$, a quantificação de água campo mencionada acima, é comumente usada para medir o consumo de água total real ou evapotranspiração da cultura (ET $)$, como explicam Farahani et al. (2009) e Abedinpour et al. (2012).

\section{Cálculos dos componentes da pegada hídrica}

As pegadas hídricas das culturas em estudo foram calculadas individualmente seguindo as seguintes equações: A pegada hídrica verde do processo de crescimento de uma cultura foi calculada dividindo-se o consumo de água verde da cultura (Cverde, $\mathrm{m}^{3} / \mathrm{ha}$ ) pela sua produtividade $(\mathrm{Y}, \mathrm{t} / \mathrm{ha}): P H_{\text {verde }}=\frac{N C H_{\text {verde }}}{P}$, em que $\mathrm{PH}_{\text {verde }}$ foi calculada pelo acúmulo de evapotranspiração da cultura (ETc) diária durante o período de crescimento completo, pelo método do balanço hídrico do solo e pelo software Crowater.

A ETc foi estimada com base em intervalos de dias ao longo de todo o período de crescimento utilizando a precipitação efetiva (Peff). A evapotranspiração de Água verde (ETverde) foi calculada como o mínimo entre os valores da evapotranspiração total da cultura (ETc) e a precipitação efetiva (Peff), isto é: $E T_{\text {verde }}=\min \left(E T_{c}\right.$, Peff $)$.

A evapotranspiração total de água verde será obtida através da soma de todas as $\mathrm{ET}_{\text {verde }}$ ao longo de todo o período de crescimento. Conforme demonstrado, foi calculada a evapotranspiração de cultura verde aplicando o fator 10 que visa converter a profundidade da água em milímetros em volume de água por superfície terrestre, em $\mathrm{m}^{3}$. ha ${ }^{-1}$. O somatório é feito ao longo do período, desde o dia do plantio $(\mathrm{d}=1)$ até o dia da colheita. Tem-se: $N \mathrm{NH}_{\text {verde }}=10 \sum_{d=1}^{d=L} E T_{\text {verde }}$, em que: $\mathrm{NHC}_{\text {verde }}=$ Necessidade hídrica de água verde $\left(\mathrm{m}^{3}\right.$ $\left.\mathrm{ha}^{-1}\right) ; \mathrm{ET}_{\text {verde }}=$ Evapotranspiração de água verde $\left(\mathrm{mm} \mathrm{dia}^{-1}\right) ; \mathrm{Dpc}=$ duração do período de crescimento (dias); $\alpha=$ fator de conversão; e $d$ = dia do plantio.

A pegada hídrica azul da cultura foi calculada dividindo-se a necessidade hídrica azul da cultura $\left(\mathrm{m}^{3}\right.$ $\mathrm{t}^{-1}$ ) pela sua produtividade $\left(\mathrm{t} \mathrm{ha}^{-1}\right)$, conforme a equação $P H_{a z u l}=\frac{N C H_{a z u l}}{P}$, em que: NCHazul foi calculada pela evapotranspiração total ao longo do ciclo da cultura. A produtividade foi obtida através do produto entre a massa fresca da parte aérea de cada planta e a população de plantas $\left(\mathrm{Kg} \mathrm{ha}^{-1}\right)$. A ETc foi estimada com base em intervalos de dias ao longo de todo o período de crescimento utilizando a precipitação efetiva (Peff). A evapotranspiração de água azul (ETazul) foi calculada a partir do máximo da equação abaixo: $\mathrm{ET}_{\mathrm{azul}}=\max (0$, $\left.E T_{c}-P_{\text {eff }}\right)$.

De acordo com a equação anterior, quando a precipitação efetiva é maior que a evapotranspiração da cultura, a $\mathrm{ET}_{\text {azul }}$ é igual à zero. A evapotranspiração total de água azul foi obtida através da soma de todas as $\mathrm{ET}_{\text {azul }}$ ao longo de todo o período de crescimento. Conforme demonstrará a próxima equação, foi calculado o consumo azul em que a evapotranspiração de cultura azul aplicando o fator 10: $N C H_{a z u l}=10 \sum_{d=1}^{d=L} E T_{a z u l}$, em que: $\mathrm{NHC}_{\text {azul }}=$ Necessidade hídrica de água azul $\left(\mathrm{m}^{3} \mathrm{ha}^{-1}\right) ; \mathrm{ET}_{\mathrm{azul}}=$ Evapotranspiração de água azul (mm dia$\left.{ }^{1}\right) ; \mathrm{Dpc}=$ duração do período de crescimento (dias); $\alpha$ = fator de conversão; e $d$ = dia do plantio. $\mathrm{A}$ NHC $\mathrm{azul}_{\text {al }}$ 
representa o total de água irrigada evaporada da área. Dessa forma, a pegada hídrica azul do cultivo de uma cultura refere-se à evapotranspiração da água irrigada apenas na área da cultura.

A pegada hídrica cinza do crescimento de uma cultura $\left(\mathrm{PH}_{\text {cinza }}\right)$ foi calculada como a taxa de aplicação de químicos por hectare (TAQ por $\mathrm{kg} \mathrm{ha}^{-1}$ ) vezes a fração de escoamento, lixiviação $(\alpha)$ dividindo pela concentração máxima aceitável (Cmáx), menos a concentração natural (Cnat) para o poluente considerado e, em seguida, dividido pela produtividade da cultura $(\mathrm{Y}, \mathrm{t} / \mathrm{ha})$, conforme $P H_{\text {cinza }}=\frac{(\alpha+\mathrm{T}) /\left(C_{\max }-C_{n a t}\right)}{\mathrm{Y}}$, em que: $P H_{\text {cinza }}=$ Pegada hídrica cinza $\left(\mathrm{m}^{3} \mathrm{t}^{-1}\right) ; \alpha=$ Fração de lixiviação $\left(\mathrm{mm} \mathrm{dia}{ }^{-1}\right) ; \mathrm{TAQ}=$ Taxa de aplicação de químicos por hectares $\left(\mathrm{kg} \mathrm{ha}^{-1}\right) ; C_{\text {máx }}=$ Concentração máxima aceitável para o poluente considerado $\left(\mathrm{Kg} \mathrm{m}^{-3}\right)$; $C_{\text {nat }}=$ Concentração natural do corpo receptor de água $\left(\mathrm{kg} \mathrm{m}^{-3}\right) ; \mathrm{e} \mathrm{Y}=$ Produtividade da cultura $\left(\mathrm{t} \mathrm{ha}^{-1}\right)$.

\section{Pegada hídrica total do processo}

O cálculo da pegada hídrica total do processo de crescimento de cada cultura foi obtido com base na soma dos componentes verde, azul e cinza, conforme demonstra $P H_{\text {total }}=P H_{\text {verde }}+P H_{\text {azul }}+P H_{\text {cinza, }}$ em que: PHtotal $=$ Pegada hídrica total de um processo de crescimento de cultura $\left(\mathrm{m}^{3} \mathrm{t}^{-1}\right) ;$ PHverde $=$ Pegada hídrica verde $\left(\mathrm{m}^{3} \mathrm{t}^{-1}\right) ;$ PHazul $=$ Pegada hídrica azul $\left(\mathrm{m}^{3} \mathrm{t}^{-1}\right) ;$ PHcinza $=$ Pegada hídrica cinza $\left(\mathrm{m}^{3} \mathrm{t}^{-1}\right)$.

\section{RESULTADOS E DISCUSSÃO}

Os componentes da $\mathrm{PH}$ do tomateiro cultivado irrigado sob o tratamento de $100 \%$ da evapotranspiração de referência são exibidos na figura 1. No caso da PH do tomateiro submetido ao tratamento de $100 \%$ da ETo, as componentes verde, azul e cinza de todas as partes da planta são diferentes e crescentes de verde para cinza. Os valores da PH total dos frutos, parte aérea e frutos + parte aérea foram, respectivamente, 1172 , 1497 e $649 \mathrm{~m}^{3} /$ ton. Desta forma, o tomateiro irrigado com $100 \%$ da ETo, produz uma evapotranspiração de 3,9mm/dia e produtividades de 4,6; 3.6 e 8.3ton/ha para frutos, parte aérea e frutos + parte aérea, respectivamente. Assim, com o tratamento de 100\% ETo, para o tomateiro produzir uma kg de fruto são necessários 1.172 litros de água. Neste tratamento, a PH total do fruto é também inferior a parte aérea da planta e a PH da soma das duas é inferior a PH dos frutos e da parte aérea.

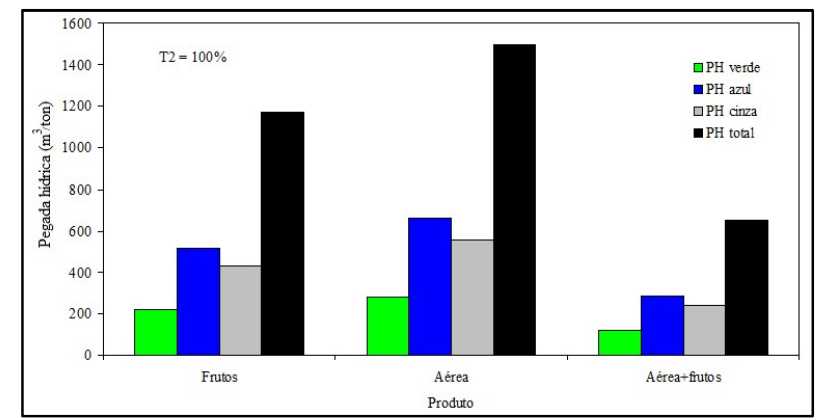

Figura 1: Componentes da pegada hídrica (PH) do tomateiro cultivado irrigado sob o tratamento de $100 \%$ da evapotranspiração de referência.

Os componentes da pegada hídrica da cultura da alface irrigada no semiárido brasileiro são exibidos na figura 2. A PH total da alface irrigada no semiárido brasileiro é baixa, de apenas $251 \mathrm{~m}^{3} /$ ton. Os 
componentes verde, azul e cinza da pegada hídrica dessa cultura são de 25, 86 e 140m³/ton, respectivamente.

A PH cinza da alface é bastante alta e representa $56 \%$ da PH total da cultura. Por outro lado, os componentes verde e azul da alface representam, respectivamente, $10 \%$ e $34 \%$ da $\mathrm{PH}$ total da cultura. A evapotranspiração média da alface na área de estudo foi de $3,7 \mathrm{~mm} /$ dia e o total de chuva durante o ciclo de cultivo foi de $120 \mathrm{~mm}$, enquanto o total irrigado nesse mesmo período foi de $142 \mathrm{~mm}$. Medeiros et al. (2012) avaliou o efeito da salinidade do solo e do manejo da fertirrigação sob a produtividade e eficiência de uso da água do tomateiro, cultivado em solo franco argiloso e em casa de vegetação. Uma das conclusões de Medeiros et al. (2012) foi que as maiores produtividades e eficiências do uso foram obtidas para o manejo de fertirrigação alternativo sob condições de baixa salinidade do solo.

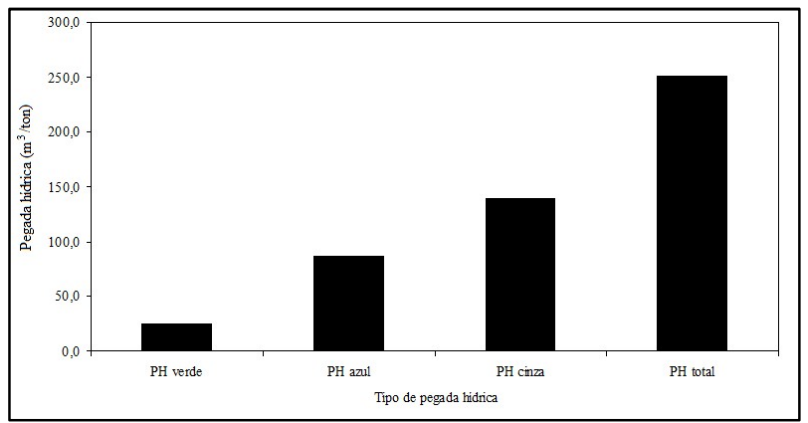

Figura 2: Componentes da pegada hídrica da alface irrigada. Legenda: $\mathrm{PH}$ verde=pegada hídrica verde; $\mathrm{PH}$ azul=pegada hídrica azul; e PH cinza=pegada hídrica cinza e $\mathrm{PH}$ total=PH verde $+\mathrm{PH}$ azul $+\mathrm{PH}$ cinza.

Os componentes da pegada hídrica da cultura do coentro irrigado no semiárido brasileiro são exibidos na figura 3. Durante o ciclo de desenvolvimento da cultura, a produtividade média foi de 0,25 ton/ha e a evapotranspiração da cultura média foi de 5,22 mm/dia. Os componentes da $\mathrm{PH}$ verde, azul e cinza foram de $232,5.000,8.207 \mathrm{~m}^{3} /$ ton, respectivamente. Desta forma, na produção de $1 \mathrm{~kg}$ de coentro são gastos 8.207 litros de água. Este alto valor da PH da cultura está associado a baixa produtividade da cultura.

A pegada hídrica cinza do coentro também é a mais alta de todas as culturas estudadas, enquanto o componente verde, que está associada à precipitação, representa apenas $2,8 \%$ da PH total. Por outro lado, os componentes azul e cinza da PH do coentro representam, respectivamente, $36 \%$ e $60 \%$ da PH total. Reis et al. (2013) determinaram ao longo do ciclo da cultura do tomateiro, a relação entre o índice de área foliar e a produtividade e, ao final do ciclo, os componentes de produção do tomateiro, em ambiente protegido.

Uma das conclusões foi que é possível determinar, em ambiente protegido, o índice de área foliar da cultura do tomateiro considerando-se os dias após o transplantio. A Figura 4 exibe os componentes da pegada hídrica do feijão irrigado na área de estudo. A menor componente é a verde e maior é azul. A PH total do feijão irrigado no semiárido brasileiro é também bastante alta. São necessários 5.507 litros de água para produzir quilo de feijão. Os componentes verde, azul e cinza da pegada hídrica dessa cultura são de 46; 3.393 e $1.666 \mathrm{~m}^{3} /$ ton, respectivamente. Portanto, a PH verde do feijão representa menos de $1 \%$ da $\mathrm{PH}$ total. 


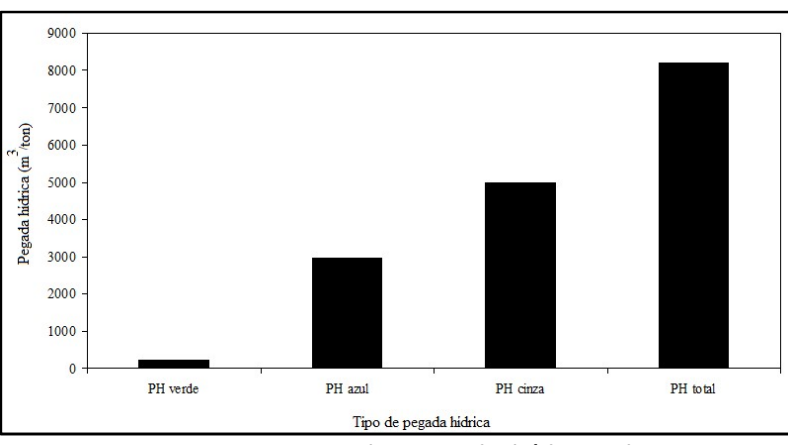

Figura 3: Componentes da pegada hídrica do coentro irrigado. Legenda: $\mathrm{PH}$ verde=pegada hídrica verde; $\mathrm{PH}$ azul=pegada hídrica azul; $\mathrm{PH}$ cinza=pegada hídrica cinza; e $\mathrm{PH}$ total=PH verde $+\mathrm{PH}$ azul $+\mathrm{PH}$ cinza.

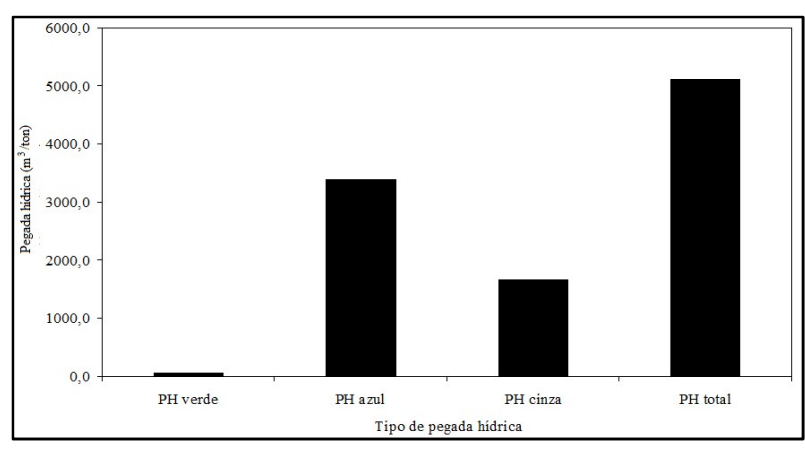

Figura 4: Componentes da pegada hídrica do feijão irrigado. Legenda: $\mathrm{PH}$ verde=pegada hídrica verde; $\mathrm{PH}$ azul=pegada hídrica azul; $\mathrm{PH}$ cinza=pegada hídrica cinza; $\mathrm{PH}$ total=PH verde $+\mathrm{PH}$ azul $+\mathrm{PH}$ cinza.

Os componentes azul e cinza do feijão representam, respectivamente, $66 \%$ e $33 \%$ da $\mathrm{PH}$ total da cultura. O alto valor da $\mathrm{PH}$ total do feijão está associado à baixa produtividade da cultura que foi de 1,2ton/ha. A evapotranspiração média do feijão na área de estudo foi de 6,2mm/dia e o total de chuva durante o ciclo de cultivo foi de apenas 5,6mm. O baixo valor da $\mathrm{PH}$ verde está associado à pluviometria na região durante o cultivo do feijão.

A figura 5 exibe os componentes da pegada hídrica da cebola irrigada no semiárido brasileiro. A PH total da cebola é bastante baixa, quando comparada com as outras culturas em análise. De acordo com os dados são necessários apenas 131 litros de água para produzir um kg de cebola. Os componentes verde, azul e cinza da pegada hídrica dessa cultura são de 14, 71 e $46 \mathrm{~m}^{3} /$ ton, respectivamente. Portanto, a $\mathrm{PH}$ verde da cebola é muita baixa, representando em torno de $10 \%$ da $\mathrm{PH}$ total. A produtividade da cebola foi de 43 ton/ha e a evapotranspiração média foi de $3,8 \mathrm{~mm} /$ dia, enquanto o total de chuva durante o ciclo de cultivo foi $92 \mathrm{~mm}$.

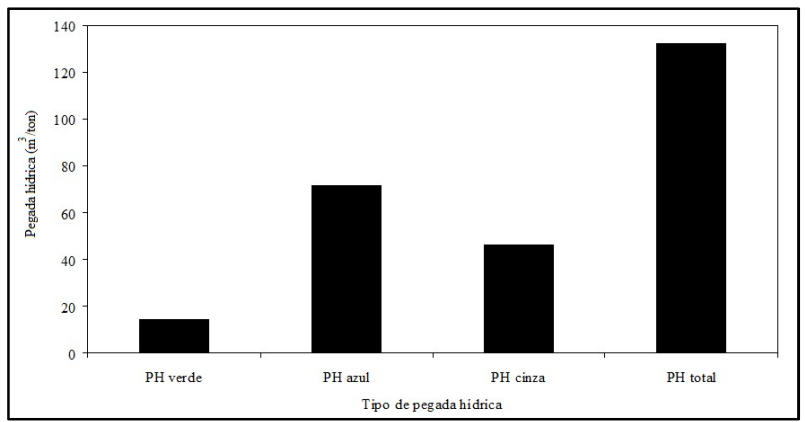

Figura 5: Componentes da pegada hídrica da cebola irrigado. Legenda: $\mathrm{PH}$ verde=pegada hídrica verde; $\mathrm{PH}$ azul=pegada hídrica azul; $\mathrm{PH}$ cinza=pegada hídrica cinza; e $\mathrm{PH}$ total=PH verde $+\mathrm{PH}$ azul $+\mathrm{PH}$ cinza

Os componentes da pegada hídrica, evapotranspiração e produtividade do tomateiro cultivado irrigado sob cinco tratamentos de irrigação no semiárido brasileiro são exibidos na tabela 1. Constata-se que a PH da parte aérea + fruto é inferior à de frutos e da parte aérea + frutos em todos os tratamentos de irrigação. Isso ocorre pelo fato que é utilizada a mesma quantidade de água nas três situações. A evapotranspiração e produtividade do tomateiro aumentam de acordo com o aumento da lâmina de água aplicada à cultura. Entretanto, a pegada hídrica não segue esta linearidade, haja vista que a PH do fruto do tomate é maior nos tratamentos com $20 \%$ ETo e $100 \%$ ETo. 
A PH cinza é maior nos tratamentos de $20 \%$ ETo, $40 \%$ ETo e a $60 \%$ ETo, enquanto nos tratamentos de $80 \%$ ETo e $100 \%$ ETo a PH azul é maior de que todos as componentes. Entretanto, apesar disso, a PH cinza ainda continua sendo muito alta e predominante no cálculo da $\mathrm{PH}$ total. Este resultado sugere que o tomateiro absorve bastante defensivos agrícolas durante todo o seu ciclo de desenvolvimento, desde a brotação até a colheita. Em todos os tratamentos de irrigação, a $\mathrm{PH}$ verde tem a menor contribuição da $\mathrm{PH}$ total, chegado a ser aproximadamente 1/3 da $\mathrm{PH}$ cinza. Este resultado sugere que irrigação tem uma contribuição mais importante do que a precipitação pluvial em face da capacidade de controle de entrada de água no sistema.

Tabela 1: Componentes da pegada hídrica, evapotranspiração e produtividade do tomateiro cultivado irrigado sob cinco tratamentos de irrigação.

\begin{tabular}{|c|c|c|c|c|c|}
\hline Tipo & Prod & $\mathrm{PH}$ verde & $\mathrm{PH}$ azul & $\mathrm{PH}$ cinza & PH total \\
\hline \multicolumn{6}{|c|}{ T1= 20\% ETo } \\
\hline Frutos & 2,7 & 200,2 & 207,4 & 724,6 & 1132,2 \\
\hline Aérea & 2,6 & 210,1 & 217,6 & 760,5 & 1188,2 \\
\hline Aérea+frutos & 5,4 & 102,3 & 106,0 & 370,4 & 578,7 \\
\hline \multicolumn{6}{|c|}{$\mathrm{T} 2=40 \%$ ETo } \\
\hline Frutos & 3,5 & 206,6 & 279,7 & 571,4 & 1057,8 \\
\hline Aérea & 2,6 & 278,2 & 376,5 & 769,2 & 1423,9 \\
\hline Aérea+frutos & 6,1 & 118,6 & 160,5 & 327,9 & 606,9 \\
\hline \multicolumn{6}{|c|}{ T3=60\% ETo } \\
\hline Frutos & 4,5 & 188,3 & 337,7 & 444,4 & 970,4 \\
\hline Aérea & 2,9 & 292,2 & 524,0 & 689,7 & 1505,8 \\
\hline Aérea+frutos & 7,3 & 116,1 & 208,2 & 274,0 & 598,2 \\
\hline \multicolumn{6}{|c|}{ T4=80\% ETo } \\
\hline Frutos & 4,4 & 218,6 & 475,9 & 454,5 & 1149,1 \\
\hline Aérea & 3,7 & 260,0 & 565,9 & 540,5 & 1366,5 \\
\hline Aérea+frutos & 8,2 & 117,3 & 255,4 & 243,9 & 616,6 \\
\hline $3,9 \mathrm{~mm} / \mathrm{dia}$ & & & & & \\
\hline \multicolumn{6}{|c|}{ T5= $100 \%$ ETo } \\
\hline Frutos & 4,6 & 219,8 & 517,4 & 434,8 & 1172,0 \\
\hline Aérea & 3,6 & 280,8 & 661,1 & 555,6 & 1497,5 \\
\hline Aérea+frutos & 8,3 & 121,8 & 286,8 & 241,0 & 649,5 \\
\hline
\end{tabular}

Os componentes da pegada hídrica das cinco culturas cultivadas irrigadas no semiárido brasileiro calculadas pelo método do balanço hídrico do solo e pelo modelo CROPWAT são exibidos na tabela 2 . A pegada hídrica total dessas culturas é também comparada com a pegada hídrica média global, conforme Mekonnen et al. (2011).

Constata-se que a PH calculada pelo modelo CROPWAT subestima os valores da PH pelo método do balanço hídrico do solo no caso de todas as culturas. A PH cinza é a maior componente nas culturas do feijão, coentro e alface, enquanto a $\mathrm{PH}$ verde é a menor componente em todas as culturas. Apenas no caso da cultura do feijão, os valores da $\mathrm{PH}$ total pelos métodos do CROPWAT e BH apresentam diferença estatisticamente significativa pelo método t-Student a nível de 5\% de probabilidade. Por outro lado, a $\mathrm{PH}$ total e as componentes verde azul e cinza das outras culturas não apresentam diferença estatisticamente significativa entre valores da ETc obtidos pelo método do BH ou pelo modelo CROPWAT.

Os valores obtidos para a pegada hídrica total das culturas analisadas são similares àquelas da pegada hídrica média global obtidas por Mekonnen et al. (2011), exceto no caso da cultura do tomate, cuja diferença produz um erro de $67 \%$. Por outro lado, no caso da cultura de feijão a diferença entre as PHs deste estudo e da média global produz um erro em torno de apenas $1 \%$. No caso das culturas do coentro e alface os erros 
também são desprezíveis. Os valores da PH do coentro obtidos no presente estudo e a média global obtidas por Mekonnen et al. (2011) foram, respectivamente, de 8.280 e $8.207 \mathrm{~m}^{3} /$ ton. Por outro lado, os valores da PH da alface obtidos no presente estudo e a média global obtidas por Mekonnen et al. (2011) foram, respectivamente, de 237 e $267 \mathrm{~m}^{3} /$ ton.

Tabela 2: Componentes da pegada hídrica $\left(\mathrm{m}^{3} / \mathrm{ton}\right)$ de cinco culturas cultivadas no semiárido brasileiro calculadas pelo método do balanço hídrico do solo, pelo modelo CROPWAT.

\begin{tabular}{|c|c|c|c|c|c|}
\hline Tipo & $\mathrm{PH}$ verde & $\mathrm{PH}$ azul & $\mathrm{PH}$ cinza & PH Total (Média global) * & PH total \\
\hline & & Tomate & & & \\
\hline $\mathrm{BH}$ & 122 & 287 & 241 & 214 & 650 \\
\hline \multirow[t]{2}{*}{ CROPWAT } & 86 & 252 & 241 & & 579 \\
\hline & & Feijão & & & \\
\hline $\mathrm{BH}$ & 47 & 3394 & 1667 & 5053 & 5107 \\
\hline \multirow[t]{2}{*}{ CROPWAT } & 47 & 1825 & 1667 & & 3538 \\
\hline & & Cebola & & & \\
\hline $\mathrm{BH}$ & 14 & 72 & 46 & 272 & 132 \\
\hline \multirow[t]{2}{*}{ CROPWAT } & 12 & 58 & 46 & & 116 \\
\hline & & Coentro & & & \\
\hline $\mathrm{BH}$ & 232 & 2975 & 5000 & 8280 & 8207 \\
\hline \multirow[t]{2}{*}{ CROPWAT } & 134 & 2100 & 5000 & & 5600 \\
\hline & & Alface & & & \\
\hline $\mathrm{BH}$ & 32 & 95 & 140 & 237 & 267 \\
\hline CROPWAT & 25 & 86 & 140 & & 252 \\
\hline
\end{tabular}

Fonte: Mekonnen et al. (2011). Legenda: *Pegada hídrica média global.

Ainda de acordo com esta tabela, a pegada hídrica verde diminui à medida em que ocorre acréscimo na lâmina de irrigação, enquanto a pegada hídrica azul não segue um padrão definido. Em estudo realizado para comparar as medições da pegada hídrica da cana-de-açúcar derivadas pelo método tradicional utilizado na sua estimativa usando o balanço hídrico completo no solo em condições de campo e o modelo CROPWAR, Silva et al. (2015) observou que pegada hídrica da cana-de-açúcar obtida com base no modelo CROPWAT superestima os valores da pegada hídrica verde e azul e subestima os valores da pegada hídrica cinza obtidos com base no balanço hídrico do solo.

A pegada hídrica cinza apresenta a menor contribuição da pegada hídrica total e seus valores decrescem em função do aumento da irrigação. Em estudo para determinar a pegada hídrica e análise econômica da cultura de alface cultivada sob irrigação no município de Itabaiana (SE), Santos (2017) concluiu que a pegada hídrica verde diminui à medida que ocorre acréscimo na lâmina de irrigação, enquanto que a pegada hídrica azul aumenta em função do aumento na lâmina de irrigação e que pegada hídrica da alface cultivado em Itabaiana (SE), obtida com base no modelo subestima os valores da PH obtida com o balanço hídrico do solo. Neste estudo, Santos (2017) observou, ainda, que a necessidade hídrica azul é a que mais contribui com o valor total da necessidade hídrica da cultura no período verão-outono

O sumário das principais características climáticas e agrometeorológicas das culturas da alface, coentro, cebola, feijão e tomate cultivadas irrigadas no semiárido brasileiro é exibido na tabela 3. Apesar de ciclo curto, a cultura do coentro tem o maior consumo hídrico e a maior pegada hídrica de todas as culturas analisadas, de $8.207 \mathrm{~m}^{3} /$ ton. A cultura com a segunda maior pegada hídrica é o feijão, com $5.507 \mathrm{~m}^{3} /$ ton, que está associada a alta componente azul da PH dessa cultura que foi a maior lâmina de irrigação, de $321 \mathrm{~mm}$, e também a componente verde da PH que está relacionada com a mais alta taxa de precipitação, que foi de $213 \mathrm{~mm}$ 
Tabela 3: Sumário das principais características climáticas e agrometeorológicas das culturas analisadas no estudo.

\begin{tabular}{|c|c|c|c|c|c|c|}
\hline Cultura & ETc & $\mathrm{Kc}$ & ETo & Prec & $\mathrm{PH}$ total & Irrig \\
\hline Alface & 3,3 & 0,78 & 6,0 & 73,4 & 650 & 205,7 \\
\hline Coentro & 5,4 & 0,90 & 4,8 & 22,0 & 8.207 & 247,8 \\
\hline Cebola & 3,6 & 0,82 & 3,6 & 92,7 & 132 & 294,5 \\
\hline Feijão & 3,8 & 1,10 & 3,7 & 213,0 & 5.107 & 321,3 \\
\hline Tomate & 3,9 & 1,06 & 4,2 & 200,6 & 267 & 309,1 \\
\hline
\end{tabular}

Legenda: ET=evapotranspiração da cultura (mm/dia); Kc=coeficiente de cultivo (adimensional); Prec.=precipitação pluvial (mm); PH total=pegada hídrica total ( $\mathrm{m}^{3} /$ ton); Irrig. =irrigação $(\mathrm{mm})$

As culturas com os maiores coeficientes de cultivo são o feijão e o tomate, com valores de 1,10 e 1,06, respectivamente. Já a maior taxa de evapotranspiração, foi da cultura da alface, e a menor foi do feijão. Silva et al. (2016), analisando o consumo hídrico da cultura do feijão caupi, cultivar potiguar, observaram que o consumo hídrico da cultura na fase de desenvolvimento vegetativo foi $159 \mathrm{~mm}$, enquanto para todo o ciclo o consumo foi $400,1 \mathrm{~mm}$.

Além disso, que a evapotranspiração média foi de 5,07mm/dia e o coeficiente de cultivo foi de 0,92. Por outro lado, Silva et al. (2018), em estudo para avaliar o efeito da data de plantio sobre a evapotranspiração da cultura (ETc), o coeficiente de cultura (Kc) do coentro, também cultivado irrigado em Itabaiana, SE, constataram que os valores médios de ETc e o Kc do coentro foram 139,8mm e 0,87, respectivamente. A demanda hídrica da cultura foi maior durante o verão e menor no inverno; e, no entanto, a sua produtividade é maior no outono e menor no inverno.

Também em experimento com plantas hortícolas, Silva et al. (2018), em estudo com a cultura da alface, constataram que essa cultura apresentou evapotranspiração média de 3,5mm/dia e coeficiente de cultivo médio de 0,82. Ainda em estudos com hortícolas, Freitas (2017) encontrou coeficiente de cultivo médio para a cultura do tomate de 0,78 ; enquanto Tavares (2016) encontrou valores do coeficiente de cultivo da alface e coentro de 0,82 e 0,86 , respectivamente.

\section{CONCLUSÕES}

O estudo realizado com cinco culturas hortícolas na Estação Agrometeorológica Experimental (EstAgro) da Universidade Federal de Campina Grande (UFCG), como parte do projeto PRONEX (Programa de Apoio aos Núcleos de Excelência), financiado pela Fapesq/CNPq, é possível extrair as seguintes conclusões: As hortaliças folhosas, como coentro e alface, têm alto componente da PH cinza comparada com os componentes verde e azul da cultura; O modelo CROPWAT pode ser utilizado com razoável precisão para o cálculo da pegada hídrica de culturas; As pegadas hídricas azul e verde aumentam de acordo com o aumento da irrigação e precipitação pluvial, porém a pegada hídrica cinza varia apenas em função da produtividade.

Além disso, a pegada hídrica calculada pelo modelo CROPWAT subestima os valores da pegada hídrica pelo método do balanço hídrico do solo nas condições edafoclimáticas do semiárido brasileiro. Os valores da pegada hídrica média global de culturas apresentadas por Mekonnen et al. (2011) não apresentam precisão satisfatória em todas as culturas analisadas neste estudo. As culturas de feijão e coentro têm alta sensibilidade a defensivos agrícolas, pesticidas, praguicidas durante todo o ciclo de desenvolvimento, desde a brotação até a colheita em face dos altos valores de suas PHs cinza. 


\section{REFERÊNCIAS}

ABABAEI, B.; ETEDALI, H. R.. Estimation of water footprint components of Iran's. wheat production: comparison of global and national scale estimates. Environment Process, v.1, p.193-205, 2014.

AHMED, S. M.; RIBBE, L.. Analysis of water footprints of rained and irrigated crops in Sudan. Journal Natural Resources, v.3, p.20-28, 2011.

ALDAYA, M. M.; HOEKSTRA, A. Y.. The water needed for Italians to eat pasta and pizza. Agricultural Systems, v.103, p.351-360, 2010.

BOMFIM, J. M. S.. Pegada hídrica e desempenho econômico da cultura do coentro (Coriandrum sativum $L$.) no agreste Sergipano. Dissertação (Mestrado em Meio Ambiente) Universidade Federal de Sergipe, São Cristóvão, 2017.

CHAPAGAIN, A. K.; HOEKSTRA, A. Y.. The global component of freshwater demand and supply: An assessment of virtual water flows between nations as a result of trade in agricultural and industrial products. Water International, v.33, p.19-32, 2008.

DABROWSKI, J. M.; MURRAY, K.; ASHTON, P. J.; LEANER, J. J. Agriculture impacts on water quality and implications for virtual water trading decisions. Ecological Economics, v.68, p.1074-1082, 2011.

GHEEWALA, S. H.; SILALERTRUKSA, T.; NILSALAB, P.; MUNGKUNG, R.; PERRET, S. R.; CHAIYAWANNAKARN, N.. Water footprint and impact of water consumption for food, feed, fuel crops production in Thailand. Water, v.6, p.16981718, 2014.

GOMES, A. R. M. G.; DAVILA, J. H. T.; GONDIM, R. S.; BEZERRA, C. B.; BEZERRA, F. M. L.. Estimativa da evapotranspiração e coeficiente de cultivo da Heliconia psittacorum $L \times$ H. spathocircinada (Arist) cultivada em ambiente protegido. Ciência Agronômica, v.37, n.1, p.13-18, 2006.

HAROU, J.; VELAZQUEZ, P.; ROSENBERG, D. E.; AZUAR, J. M.; LUND, D. J.; HOWITT, D. R.. Hydro-Economic Models: Concepts, Design, Applications, and Future Prospects. Journal of Hydrology, v.375, n.4, p.627-643, 2009.

HOEKSTRA, A. Y.; ALDAYA, M. M. C. A. K.; MEKONNEN, M. M.. The Water Footprint Assessment Manual: Setting the Global Standard. Abingdon: Routledge, 2011.

LAMASTRA, L.; SUCIU, N. A.; NOVELLI, E.; TREVISAN, M.. A new approach to assessing the water footprint of wine: an Italian case study. Science of the Total Environmental, v.490, p.748-756, 2014.

LEVIDOW, L.; ZACCARIA, D.; MAIA, R.; VIVAS, E.; TODOROVIC, M.; SCARDIGNO, A.. Improving water-efficient irrigation: Prospects and difficulties of innovative practices. Agricultural Water Management, v.146, p.84-94, 2014.

MEDEIROS, P. R. F.; DUARTE, S. N.; SILVA, E. F. F.. Eficiência do uso de água e de fertilizantes no manejo de fertirrigação no cultivo do tomateiro sob condições de salinidade do solo.
Revista Brasileira de Ciências Agrárias, v.7, n.2, p.344-351, 2012.

MEKONNEN, M. M.; HOEKSTRA, A. J.. The green, blue and grey water footprint of cropsand derived crop products. Hydrology and Earth System Sciences, v.15, p.1577-1600, 2011.

MEKONNEN, M. M.; HOEKSTRA, A. Y.. A global assessment of the water footprint of farm animal products. Ecosystems, v.15, p.401-415, 2012.

MEKONNEN, M. M.; HOEKSTRA, A. Y.. Four billion people facing severe water scarcity. Sciences Advances, v.2, p.1-6, 2010.

NANA, E.; CORBARI, C.; BOCCHIOLA, D.. A model for crop yield and water footprint assessment: study of maize in the Povalley. Agricultural System, v.127, p.139-149, 2014.

REIS, L. S.; AZEVEDO, C. A. V.; ALBUQUERQUE, A. W; SILVA JUNIOR, J. F.. Índice de área foliar e produtividade do tomate sob condições de ambiente protegido. Revista Brasileira de Engenharia Agrícola e Ambiental, v.17, n.4, p.386-391, 2013.

RIBEIRO, C. S.. Pegada hídrica e água virtual: estudo de caso da manga no submédio do Vale do São Francisco, Brasil. Tese (Doutorado em Economia) - Universidade Federal da Bahia, Salvador, 2014.

ROCHA, S. R.; STUDART, T. M. C.. A pegada hídrica das exportações agrícolas de Pernambuco. Revista de Geografia, v.31, n.3, p.190-210, 2014.

SANTIAGO, A. D.; CHICO, D.; ANDRADE JUNIOR, A. S.; GARRIDO, A.; CARNAÚBA, P. J. P.. Pegada hídrica da cana-deaçúcar e etanol produzidos no estado de Alagoas, Brasil. Agrometeoros, v.25, n.1, p.209-216, 2017.

SANTOS, K. S.. Pegada hídrica e análise econômica da cultura de alface cultivada sob irrigação no município de Itabaiana - SE. Dissertação (Mestrado em Meteorologia) Universidade Federal de Campina Grande, Campina Grande, 2017.

SILVA, V. P. R.; ALBUQUERQUE, M. F.; ARAÚJO, L. E.; CAMPOS, J. H. B.; GARCÊZ, S. L. A.; ALMEIDA, R. S. R. Medições e modelagem da pegada hídrica da cana-deaçúcar cultivada no Estado da Paraíba. Revista Brasileira de Engenharia Agrícola e Ambiental, v.19, n.6, p.521-526, 2015.

SILVA, V. P. R.; ALEIXO, D. O.; DANTAS, J. N.; MARACAJÁ, K. F. B.; ARAÚJO, L.. Uma medida de sustentabilidade ambiental: Pegada hídrica. Revista de Engenharia Agrícola e Ambiental, v.17, p.100-105, 2013.

SILVA, V. P. R.; SILVA, B. B.; BEZERRA, J. R. C.; ALMEIDA, R. S. R.. Consumo hídrico e viabilidade econômica da cultura do feijão cupi cultivada em clima semiárido. Irriga, v.21, n.4, p.662-672, 2016. 
SILVA, V. P. R.; SOUSA, I. F.; TAVARES, A. L.; SILVA, T. G. F.; SILVA, B. B.; HOLANDA, R. M.; BRITO J. I. B.; BRAGA, C. C.; SOUZA, E. P.; SILVA, M. T.. Evapotranspiration, crop coefficient and water use efficiency of coriander grown in tropical environment. Horticultura Brasileira, v.36, n.4, p. 446-452, 2018.
YOO, S. H.; KIM, T.; IM, J. B.; CHOI, J. Y.. Estimation of the international virtual water flow of grain crop products in Korea. Paddy Water Environment, v.10, p.83-93, 2012.

A CBPC - Companhia Brasileira de Produção Científica (CNPJ: 11.221.422/0001-03) detém os direitos materiais desta publicação. Os direitos referem-se à publicação do trabalho em qualquer parte do mundo, incluindo os direitos às renovações, expansões e disseminações da contribuição, bem como outros direitos subsidiários. Todos os trabalhos publicados eletronicamente poderão posteriormente ser publicados em coletâneas impressas sob coordenação da Sustenere Publishing, da Companhia Brasileira de Produção Científica e seus parceiros autorizados. Os (as) autores (as) preservam os direitos autorais, mas não têm permissão para a publicação da contribuição em outro meio, impresso ou digital, em português ou em tradução. 